\title{
Characteristics of the Taylor microscale in the solar wind/foreshock: magnetic field and electron velocity measurements
}

\author{
C. Gurgiolo ${ }^{1}$, M. L. Goldstein ${ }^{2}$, W. H. Matthaeus ${ }^{3}$, A. Viñas ${ }^{2}$, and A. N. Fazakerley ${ }^{4}$ \\ ${ }^{1}$ Bitterroot Basic Research, Hamilton, MT, USA \\ ${ }^{2}$ Heliospheric Physics Laboratory, Code 672, NASA Goddard Space Flight Center, Greenbelt, MD, USA \\ ${ }^{3}$ Bartol Research Foundation, University of Delaware, Newark, DE, USA \\ ${ }^{4}$ Mullard Space Science Laboratory, University College London, Holmbury St. Mary Dorking, Surrey RH5 6NT, UK
}

Correspondence to: C. Gurgiolo (chris@gurgiolo.com)

Received: 3 December 2012 - Revised: 18 October 2013 - Accepted: 23 October 2013 - Published: 22 November 2013

\begin{abstract}
The Taylor microscale is one of the fundamental turbulence scales. Not easily estimated in the interplanetary medium employing single spacecraft data, it has generally been studied through two point correlations. In this paper we present an alternative, albeit mathematically equivalent, method for estimating the Taylor microscale $\left(\lambda_{\mathrm{T}}\right)$. We make two independent determinations employing multi-spacecraft data sets from the Cluster mission, one using magnetic field data and a second using electron velocity data. Our results using the magnetic field data set yields a scale length of $1538 \pm 550 \mathrm{~km}$, slightly less than, but within the same range as, values found in previous magnetic-field-based studies. During time periods where both magnetic field and electron velocity data can be used, the two values can be compared. Relative comparisons show $\lambda_{\mathrm{T}}$ computed from the velocity is often significantly smaller than that from the magnetic field data. Due to a lack of events where both measurements are available, the absolute $\lambda_{\mathrm{T}}$ based on the electron fluid velocity is not able to be determined.
\end{abstract}

Keywords. Interplanetary physics (Plasma waves and turbulence)

\section{Introduction}

Turbulence is one of the most efficient means of mixing and transporting energy, momentum, and mass in fluid systems, which includes the solar wind. Figure 1 is a generic picture of the turbulence cascade showing the three basic turbulent ranges as well as the location of three fundamental scales. Most of the turbulent energy is contained in the energy-containing range that comprises the lowest frequencies and the largest eddy structures. The eddies are inherently unstable and the break in the spectrum labeled as the correlation scale is essentially the size of the largest eddy in the system. The inertial range covers the central portion of the spectrum and includes eddy sizes between the largest and those that begin to be influenced by dissipation. Here inertial or inviscid processes dominate the cascade in energy or, equivalently, the eddy size. Below the inertial range is the dissipative range where eddy energy dissipates as heat ${ }^{1}$. The dissipation range is characterized by a change in spectral index at what is known as the Kolmogorov scale (see below), which is the smallest supported eddy size, beyond which the eddies are overdamped. For a viscous fluid, just above the Kolmogorov scale lies the third fundamental scale, the Taylor microscale. Evaluation of this length scale in the solar wind is the main topic of the present paper.

While the solar wind is not technically a fluid, it behaves as such above the dissipation scale and the use of an MHD formalism and terminology is often adopted in the discussion and study of the turbulence there. Eddies in the solar wind are probably best thought of as semi-coherent or semi-organized regions of energy, and while viscosity and resistivity exist they are certainly nonclassical, being

\footnotetext{
${ }^{1}$ Note that the solar wind is a low-collisionality medium having numerous possible kinetic dissipation mechanisms at scales smaller than the ion inertial length, where the spectrum is observed to steepen. Here we will assume that the "steepening" corresponds to the end of the inertial range, and therefore employ simpler hydrodynamic/viscous terminology in describing the Taylor microscale. See Matthaeus et al. (2008) for more discussion of this point.
} 


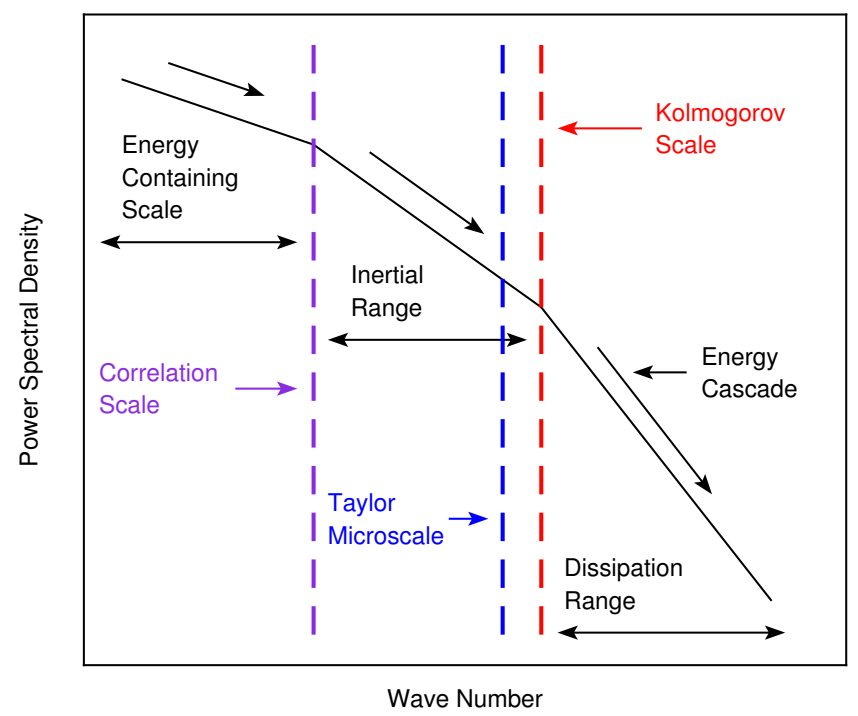

Fig. 1. Generic picture of turbulence in the solar wind showing the primary turbulence ranges and scale lengths.

implemented through wave-particle interactions rather than collisions (Holzer et al., 1986; Verma, 1996). The fact that turbulence in the inertial range in the solar wind generally exhibits Kolmogorov or Kraichnan characteristics (Podesta et al., 2007; Salem et al., 2009, 2012) is indicative of the validity of the use of fluid hydrodynamics to describe the solar wind and its environs. $\lambda_{\mathrm{T}}$ was first described by Taylor (1935) using a fluid-based formalism. For Kolmogrov scaling, $\lambda_{\mathrm{T}}$ is within the inertial range, located at $2 / 3$ the distance between the inertial and dissipation scales (measured from the inertial scale).

Multiple spacecraft estimation (Matthaeus et al., 2005) gives values of the correlation scale $\lambda_{\mathrm{c}} \approx 1.3 \times 10^{11} \mathrm{~cm}$, or $0.008 \mathrm{AU}$. The magnetic inertial range power law terminates with subsequent steepening at frequencies near $0.5 \mathrm{~Hz}$ at $1 \mathrm{AU}$. For solar wind speeds of $V_{\mathrm{sw}} \sim 300-500 \mathrm{~km} \mathrm{~s}^{-1}$ this corresponds to spatial scales of 600 to $1000 \mathrm{~km}$, a few times the ion inertial scale $\mathrm{c} / \omega_{p i} \approx 228 / \sqrt{n} \mathrm{~km}$, where $n$ is density in $\mathrm{cm}^{-3}$ (Leamon et al., 1998). This inner scale, or scale at which the inertial range terminates, is often described as a "dissipation scale", in analogy with hydrodynamic terminology, even though we do not know in detail what mechanisms are responsible for dissipation in the solar wind (Barnes, 1979; Tu and Marsch, 1995; Borovsky, 2004).

It is convenient to introduce the Taylor microscale in the context of a viscous dissipation function, as in hydrodynamics. Using brackets $(<\ldots>)$ to represent the ensemble average, for a typical speed $u=\sqrt{\left\langle\left|v_{\mathrm{f}}\right|^{2}>\right.}$, fluid velocity $v_{\mathrm{f}}$, mean square vorticity $\Omega=<\left|\nabla \times v_{\mathrm{f}}\right|^{2}>$, characteristic vorticity $\omega=\sqrt{\Omega}$, and viscosity $v$, we readily find from the Navier-Stokes equations that the rate of decay of energy (absent of forcing) is $\mathrm{d} u^{2} / \mathrm{d} t=-v \omega^{2} \equiv-\varepsilon$. To write the decay rate in terms of energy, we may define the Taylor microscale
$\lambda_{\mathrm{T}}$ by the relation

$\omega^{2}=\frac{u^{2}}{\lambda_{\mathrm{T}}^{2}}$.

Suppose we introduce a correlative (outer) scale $\lambda_{\mathrm{c}}$, largescale Reynolds number $R \equiv \frac{u \lambda_{c}}{v}$. Then, by dimensional analysis, a characteristic dissipation scale (Kolmogorov of inner scale) is $\lambda_{\mathrm{d}}=\left(\frac{v^{3}}{\varepsilon}\right)^{\frac{1}{4}}$ so that $\lambda_{\mathrm{d}}=\lambda_{\mathrm{c}} R^{-\frac{3}{4}}$. One readily finds also that $\lambda_{\mathrm{T}} / \lambda_{\mathrm{c}}=R^{-\frac{1}{2}}$. It is clear that for viscous hydrodynamics one expects to always have $\lambda_{\mathrm{T}}>\lambda_{\mathrm{d}}$.

In turbulence analysis one often works with correlation functions such as $R_{i j}(r)=<u_{i} u_{j}>$ or its trace $R(r)=<$ $u \cdot u^{\prime}>$, where the prime denotes an offset by a spatial lag $\boldsymbol{r}$. It is then straightforward to see that $\omega^{2}=-R^{\prime \prime}(0)=u^{2} / \lambda_{\mathrm{T}}^{2}$, and then from this relation that the Taylor scale also enters in the expansion of the second-order structure function $S_{2}(r)=<\left|v_{\mathrm{f}}-v_{\mathrm{f}}^{\prime}\right|^{2}>=u^{2} \hat{S}_{2}(r)$ for small spatial separations $\mathrm{r}$. In particular, when $S_{2}$ is regular at the origin, $S_{2}(r) \approx 1-\frac{r^{2}}{\lambda_{\mathrm{T}}^{2}}$ for small $r<<\lambda_{\mathrm{d}}$. This expansion forms one basis for measurement of the Taylor scale (Matthaeus et al., 2005; Weygand et al., 2007). Another approach, which we exploit here, is to directly estimate the mean square curl, employing the relation $\omega^{2}=u^{2} / \lambda_{\mathrm{T}}^{2}$. Below we will apply these ideas to the magnetic field and the electron fluid velocity to compute their respective Taylor microscales.

Although determining $\lambda_{\mathrm{T}}$ is fairly routine for fluid turbulence (Kailasnath and Sreenivasan, 1993; Belmabrouk and Michard, 1998; Segalini et al., 2011), due to limitations in the cadence of plasma instruments and accessibility to multispacecraft data sets, $\lambda_{\mathrm{T}}$ had not been evaluated in space plasmas before the work of Matthaeus et al. (2005). Subsequent work (Weygand et al., 2007, 2009, 2010, 2011) has explored the size and symmetry properties of $\lambda_{\mathrm{T}}$ in both the solar wind and magnetosphere. As with the other turbulence-related fundamental scale lengths, it can be estimated from either the magnetic field or plasma velocity. As one of the three basic lengths that characterize turbulence, one would expect that no matter how it is measured, the results should be similar. This is not clear, however, and as of yet has not been directly tested. It is well known that in the solar wind the magnetic field and velocity turbulence spectra often exhibit different spectral indices in the inertial range: $-5 / 3$ and $-3 / 2$, respectively (Podesta et al., 2007; Salem et al., 2009). The spectra, however, do not necessarily convey information about $\lambda_{\mathrm{T}}$.

As noted above, there are two approaches that can be used to estimate $\lambda_{\mathrm{T}}$. The first was proposed by Matthaeus et al. (2005) and implemented by Matthaeus et al. $(2005,2008)$ and Weygand et al. (2007, 2009, 2011, 2010). This approach consists of estimating the radius of curvature of the correlation function at small separations (Taylor, 1935). Using the correlation function to estimate $\lambda_{\mathrm{T}}$ has several advantages. The primary one is that if the turbulence is assumed to be isotropic (a condition that the work of Weygand et al. (2009, 
2010) suggests may not generally be the case), then $\lambda_{T}$ can be estimated from only a pair of spacecraft. It can even be estimated from a single spacecraft if the Taylor frozen in condition (Taylor, 1938) is valid and sufficiently high-resolution data are available. The process involves several steps, which basically consist of the following:

- Application of any preconditioning to the data.

- Evaluation of the cross-correlation expression $R(r)=<\alpha(x) \cdot \alpha(x+r)>$, where $\alpha$ represents the measurement used to estimate $\lambda_{\mathrm{T}}$ (generally B or V).

- Usage of the cross-correlation expression in the small $r$ limit $\left(R(r) \approx<\alpha^{2}>\left(1-\frac{r^{2}}{a \lambda_{T_{\alpha}}^{2}}\right)+\ldots\right.$ to solve for the Taylor Microscale $\left(\lambda_{T_{\alpha}}\right)$ within a number of time periods with different separation distances. In the expression $r$ is the spacecraft separation when using the two spacecraft approach and the spatial lag when using a single spacecraft approach.

- Extrapolation of the estimated $\lambda_{\mathrm{T}}(r)$ to get $\lambda_{\mathrm{T}}(0)$.

Application of this technique using magnetic field data from both the solar wind and plasma sheet yields $\lambda_{\mathrm{T}}$ scale lengths of 2400 and $1900 \mathrm{~km}$, respectively (Weygand et al., 2007). An additional study by Weygand et al. (2011) has shown that the magnetic-field-based estimates of $\lambda_{\mathrm{T}}$ in the solar wind exhibit an anisotropy between values derived parallel $(3500 \mathrm{~km})$ and perpendicular $(1200 \mathrm{~km})$ to the mean magnetic field. The anisotropy is seen only for solar wind speeds between 450 and $600 \mathrm{~km} \mathrm{~s}^{-1}$. $\lambda_{\mathrm{T}}$ appears to be isotropic outside that range.

A second approach from which $\lambda_{\mathrm{T}}$ may be estimated is through the generalization of the exact relationship given in Eq. (1), written as

$\lambda_{\mathrm{T} \alpha}=\sqrt{\frac{<\alpha^{2}>}{<(\nabla \times \alpha)^{2}>}}$,

where $\alpha$ is the fluctuating (mean free) component of the parameter forming the basis of the estimates (e.g., fluid velocity, magnetic field) (see Taylor, 1935; Batchelor, 1953). Initial results using this approach are presented here for the first time. Although the two approaches are mathematically essentially the same, they do differ in their method of solution. In the first approach the correlation values are determined to a high accuracy by averaging over a large number of observations and then the trend in $r$ is evaluated allowing $\lambda_{\mathrm{T}}$ to be estimated at zero spacecraft separation. In the above approach, however, each interval of data supplies its own estimate of $\lambda_{\mathrm{T}}$ and the individual estimates are then combined and extrapolated to zero spacecraft separation. In essence then the order of the averaging and computing derivative is reversed between the two methods. In addition the above definition does not rely on an assumption of isotropy.
The use of Eq. (2) to estimate $\lambda_{\mathrm{T}}$ presents several challenges. Foremost among these is the estimation of the spatial gradients required to determine the curl in the denominator of Eq. (2). Estimates of the gradients require time-contiguous measurements from multiple (i.e., at least four) spacecraft, which in this paper are supplied by the Cluster spacecraft for both the magnetic field and electron plasma data sets. The time contiguous condition is covered in all telemetry modes for the magnetic field but only in burst mode for the electron data. This greatly restricts the number of occasions in which $\lambda_{\mathrm{T}}$ can be estimated using the electron fluid velocity. A second restriction placed on the estimates of the spatial gradients is that they can only be made during times when the spacecraft are in a nearly perfect tetrahedral configuration. This limits the length of time over which the ensemble averages in Eq. (2) can be formed. The two restrictions combine to limit the scale lengths over which this approach can be used to values generally below the correlation scale length. In order to overcome this we use a two-step approach in estimating $\lambda_{\mathrm{T}}$ over the correlation scale. We first compute $\lambda_{\mathrm{T}}$ over a number of different scale lengths (done by lowpass-filtering the data at different frequencies) and then use a plot of $\lambda_{\mathrm{T}}$ vs. scale length to project these values back to the correlation scale length.

In this paper we present estimates of $\lambda_{\mathrm{T}}$ in the solar wind/foreshock using Cluster data obtained between 2001 and 2009. There has been no attempt to separate foreshock from solar wind in the analysis. As this is basically a statistical analysis, and since the intervals used are not arbitrary in that we are confined to times where the spacecraft are in a reasonable tetrahedral configuration, we have merged both solar wind and foreshock intervals (and indeed most intervals contain a mixture of the two regions) to keep statistics as high as possible. These regions may be separated in a later work. We report here results using both the magnetic field and the electron fluid velocity.

\section{Instrumentation}

This study makes use of data sets from multiple Cluster experiments. $\lambda_{T}$ based on the electron fluid velocity is constructed from moments derived from the electron velocity distribution functions (VDF) acquired by the Plasma Electron And Current Experiment (PEACE). PEACE consists of a pair of hemispherical electrostatic analyzers on each of the Cluster satellites (Johnstone et al., 1997). The two analyzers, designated HEEA (high-energy electrostatic analyzer) and LEEA (low-energy electrostatic analyzer) are separated by $180^{\circ}$ on the satellite and differ only in their geometric factors (HEEA has the larger geometric factor). Despite their acronyms, both can cover the energy range 0.6 to $26 \mathrm{keV}$. The analyzers' fields of view are perpendicular to the spacecraft spin axis, i.e., approximately perpendicular to the GSE ecliptic. Each analyzer covers $180^{\circ}$ in elevation 
in 12 sectors. The full $360^{\circ}$ of azimuth is covered in one rotation of the spacecraft so that a three-dimensional snapshot of the electron distribution is accumulated once per spin $(\sim 4 \mathrm{~s})$. Data from both analyzers are used in this paper depending on which analyzer is active during the time period being analyzed.

Because of telemetry restrictions, PEACE generally returns only a subset of the total data collected onboard. Exactly what is returned depends on the instrument mode, which can be separately commanded for each analyzer on each of the spacecraft. The telemetry rate determines the frequency with which full three-dimensional distributions are downloaded. During the time intervals analyzed in this paper, all satellites were operating in burst mode telemetry and PEACE was returning 3-D distributions every four seconds. Because the data sets must be time contiguous, this is a necessary requirement and one that greatly reduces the number of available events. During these times the analyzers were generally, but not always, returning data in either the 3DXP1 data mode (26 energy steps, 32 azimuth sectors, and 6 summed elevation zones) over the approximate energy range 5.0 to $1050.0 \mathrm{eV}$ on all spacecraft, or in the 3DXP1 mode on $\mathrm{C} 1$ and $\mathrm{C} 3$ and in the 3DX1 mode (30 energy steps, 32 azimuth sectors, and 12 elevation zones over the approximate energy range 5.0 to $2550 \mathrm{eV}$ ) on $\mathrm{C} 2$ and $\mathrm{C} 4$.

Data from both the Electric Field and Waves (EFW) experiment and the Waves of High frequency and Sounder for Probing of Electron density by Relaxation (WHISPER) experiment are used in the estimation of the electron plasma moments. EFW provides the spin-averaged spacecraft potential, used to correct the energy bin edges of the PEACE energy steps. Active sounding by WHISPER distorts the spacecraft potential, which in turn affects the estimated moments. Moments computed when WHISPER is actively sounding are dropped from the analysis and the resulting temporal gaps are filled using a linear interpolation.

$\lambda_{\mathrm{T}}$ based on the magnetic field is constructed from 5vector-per-second (VPS) data provided by the Fluxgate Magnetometer (FGM) experiment through the Cluster Active Archive (CAA). When used to construct estimates of $\lambda_{T}$ in conjunction with $\lambda_{\mathrm{T}}$ estimates derived using the electron velocity, the data are averaged down to the spacecraft spin period, which keeps it at the same resolution as the moment data; otherwise $\lambda_{\mathrm{T}}$ estimates are based on the 5 VPS $(0.2 \mathrm{~s})$ data resolution.

FGM consists of two triaxial fluxgate magnetometers on each of the four Cluster spacecraft (Balogh et al., 1997). At nominal telemetry rates the experiments are able to return five magnetic field vectors per second. This allows magnetic field data $\lambda_{\mathrm{T}}$ estimates to be constructed during times when the spacecraft are not using burst mode telemetry, which greatly increases the number of events available for analysis. Statistically significant estimates of $\lambda_{\mathrm{T}}$ can be made from the 5 VPS data in time intervals as short at $15 \mathrm{~min}$; however for the current study, intervals of $70 \mathrm{~min}$ were nominally used, with none shorter than $60 \mathrm{~min}$.

\section{Analysis technique}

Estimates of $\lambda_{\mathrm{T}}$ from the electron fluid velocity or magnetic field in this paper are based on Eq. (2). The steps taken to estimate $\lambda_{\mathrm{T}}$ values basically follow those used to estimate $\lambda_{\mathrm{T}}$ from the correlation function in that one must first precondition the data to remove the mean field and then extrapolate the results to zero spacecraft separation. The dependence of the estimated $\lambda_{\mathrm{T}}$ on spacecraft separation comes from the computation of the curl in the denominator of Eq. (2) and will be discussed in more detail in Sect. 3.3.

The steps taken in estimating $\lambda_{\mathrm{T}}$ from Eq. (2) are given below in the order applied and will be described in detail in the following sections.

- When using the solar wind velocity, compute electron moments from all four spacecraft.

- Remove mean value from all data sets.

- Produce estimates of the spatial derivatives and construct estimates of $\nabla \times \alpha$.

- Produce estimates of $\lambda_{\mathrm{T}}$ from Eq. (2).

- Perform all necessary corrections for spacecraft separation and extrapolate $\lambda_{\mathrm{T}}$ to cover the full inertial range.

\subsection{Moments}

Estimating $\lambda_{\mathrm{T}}$ from the electron fluid velocity requires calculating the velocity moment from the (measured) phase space distribution. The numerical method used to estimate the electron moments has been presented in detail in Gurgiolo et al. (2010), and we refer interested readers to that publication. It should be noted that to ensure that the moments derived on each spacecraft cover the same energy range for the entire time interval analyzed, the lower energy limits used in the integrals are set to the highest lower energy measurable after correcting for the spacecraft potential, while the lower velocity limit is set to the minimum upper energy measured on the four spacecraft. For the moments to be time contiguous, all spacecraft must be returning data in burst telemetry mode. This ensures that a full 3-D distribution is being returned from each spacecraft in each spin.

\subsection{Removing the mean field}

There are multiple methods available to remove a mean field in a data set. The method employed in this study is to apply a low-pass Savitzky-Golay filter to the data and then subtract the filtered from the unfiltered data to obtain the fluctuating component. All applications of the Savitzky-Golay in 


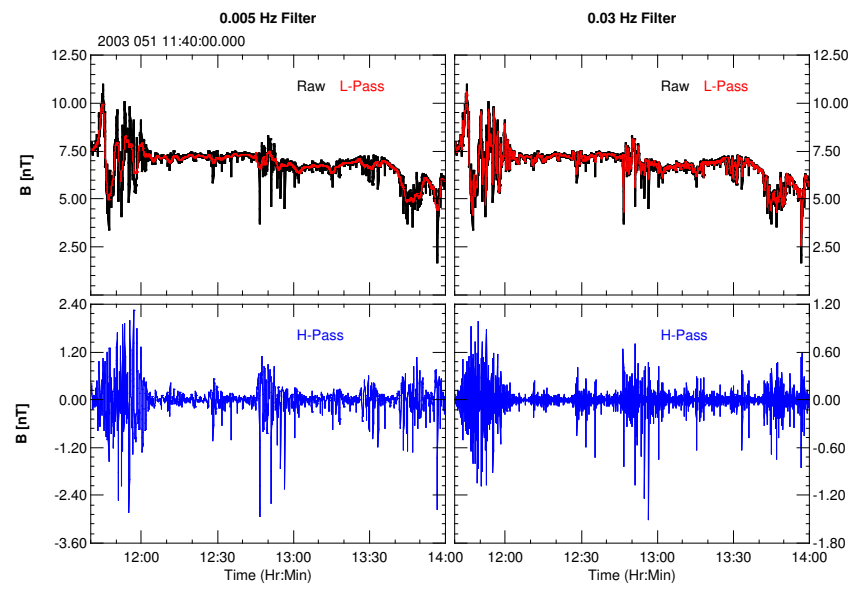

Fig. 2. Mean field removal for two Savitzky-Golay low-pass filters $(0.005$ and $0.03 \mathrm{~Hz})$. The top row of plots shows the input data (black) and the low-pass filter (red). The lower row of plots shows the high-pass data formed by subtracting the low-passed data from the input data. The high-passed data constitute the variable component of the field used in the estimation of $\lambda_{\mathrm{T}}$.

this paper fit a polynomial of degree 2 (see pages 650-655 in Press et al. (1999)). The advantages in using a SavitzkyGolay low-pass filter include that the filter can be tuned to any frequency, the filters produce negligible feature broadening in the data and only minimal changes in signal height, and the filters allow the data set to be used at its native temporal resolution. An example of the removal of the mean field is shown in Fig. 2 for the two filter frequencies of 0.005 and $0.03 \mathrm{~Hz}$.

The low-pass filter frequency establishes the scale length over which the data are processed - essentially

$\lambda=V_{\mathrm{sw}} / f$

where $\lambda$ is scale length in $\mathrm{km}, V_{\mathrm{sw}}$ is the solar wind velocity in $\mathrm{km} \mathrm{s}^{-1}$ and $f$ is the low-pass cutoff frequency in $\mathrm{Hz}$. We compute $\lambda_{\mathrm{T}}$ at eight equally logarithmically spaced cutoff frequencies ranging from 0.03125 to $0.001 \mathrm{~Hz}$. The lowest frequency that we employ is equivalent to a scale length of $\sim 5 \times 10^{5} \mathrm{~km}$, somewhat less than the $2 \times 10^{6} \mathrm{~km}$ correlation length of solar wind turbulence as determined from the upper inflection point in magnetic field spectra taken within the time period used in the study (this value is consistent with previous estimations; for example, see Matthaeus et al., 2005; Wicks et al., 2010). Using a series of filter frequencies, however, provides (as will be shown later) an extremely linear relationship between the estimated $\lambda_{\mathrm{T}}$ and the log of the low-pass cutoff frequency, which allows $\lambda_{\mathrm{T}}$ to be extended out to the correlation scale length.

\subsection{Estimates of the spatial derivatives and curl}

One of the unique features provided by the Cluster spacecraft is the ability to estimate the spatial variation of parameters measured on all spacecraft across the constellation volume. The procedure used to accomplish this and its associated limitations has already been described in detail in Gurgiolo et al. $(2010,2011)$ and is only briefly summarized here.

Spatial derivatives of a scalar quantity $Q$, such as the component of a vector, can be estimated provided that it is known at a minimum of four non-coplanar spatial locations, a condition met by the four Cluster spacecraft. With only four data points the variation of $Q$ across the volume defined by the spacecraft is provided by the set of equations

$Q_{j}=a+b X_{j}+c Y_{j}+d Z_{j}$,

where $Q$ is the quantity being fit, $j$ is the spacecraft index, $(a, b, c, d)$ are a set of unknown coefficients, and $(X, Y, Z)$ are the spacecraft position coordinates. For any $Q$, Eq. (4) expands to four equations, one per spacecraft. By way of an explicit example the equations for the quantity $Q_{x}$ are given by the coupled set of equations

$$
\begin{aligned}
& \text { C1: } Q_{x 1}=a_{x}+b_{x} X_{1}+c_{x} Y_{1}+d_{x} Z_{1} \\
& \text { C2 }: Q_{x 2}=a_{x}+b_{x} X_{2}+c_{x} Y_{2}+d_{x} Z_{2} \\
& \text { C3: } Q_{x 3}=a_{x}+b_{x} X_{3}+c_{x} Y_{3}+d_{x} Z_{3} \\
& \text { C4 } Q_{x 4}=a_{x}+b_{x} X_{4}+c_{x} Y_{4}+d_{x} Z_{4} .
\end{aligned}
$$

These can be solved using any number of common analysis techniques.

Estimates of both the divergence $(\nabla \cdot V)$ and vorticity $(\nabla \times$ $\boldsymbol{V}$ ) of any vector quantity $\boldsymbol{V}$ can be constructed from (5) as

$$
\begin{aligned}
& \nabla \cdot \boldsymbol{V}=b_{x}+c_{y}+d_{z}, \\
& \nabla \times \boldsymbol{V}=\left(c_{z}-d_{y}\right) \widehat{x}+\left(d_{x}-b_{z}\right) \widehat{y}+\left(b_{y}-c_{x}\right) \widehat{z} .
\end{aligned}
$$

Note that with the restriction to linear solutions there is no position dependence in either the divergence or curl. The curl is used in Eq. (2) in the estimation of $\lambda_{\mathrm{T}}$.

There are two explicit assumptions made in the application of Eq. (5): the measurements are independent and the variation in the parameter is linear across the spacecraft constellation. The first is generally met so long as the average travel time of the plasma across the constellation (average spacecraft separation divided by the average solar wind speed) is less than the temporal resolution of the measurement. This condition is more restrictive when using plasma parameters that have $4 \mathrm{~s}$ cadence than when using magnetic field measurements that have a $0.02 \mathrm{~s}$ cadence. The latter can be used for the most part down to spacecraft separations as small as $250 \mathrm{~km}$, while the former is generally limited to separations greater than $3500 \mathrm{~km}$.

The assumption of linearity is generally not met here. The issue is that we generally use the fluctuating component of the data obtained by subtracting out the mean. Because of this the derivatives need to be estimated using a finite-difference formalism (and not by Eq. 6 and 7), and this explicitly introduces a dependence on the spacecraft separation that will be apparent both in estimates of the divergence 
$\nabla \times$ B Estimates with B constant at $5000 \mathrm{nT}$
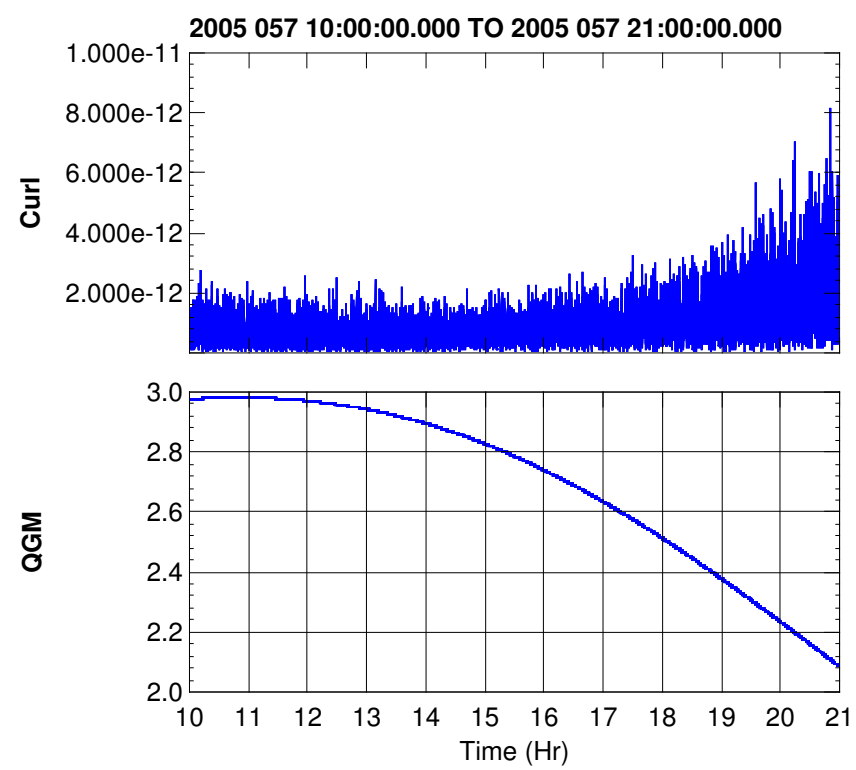

Fig. 3. Variation in the estimated curl due to variations in the Cluster configuration.

and curl, as well as in estimates of $\lambda_{\mathrm{T}}$ because of the curl in Eq. (2). A simple way to see how this dependence on spacecraft separation arises is to reduce Eq. (5) to a 1-D formulation. If there are only two spacecraft, say $\mathrm{C} 1$ and $\mathrm{C} 2$ aligned along $X$, Eq. (5) can be written as $Q_{j}=a+b X_{j}$, where $j$ is the spacecraft. The derivative $\frac{\mathrm{d} Q}{\mathrm{~d} X}$ is now estimated from

$\frac{\mathrm{d} Q}{\mathrm{~d} X}=\lim _{\Delta X \rightarrow 0} \frac{\left(Q_{1}-Q_{2}\right)}{\left(X_{1}-X_{2}\right)}=\lim _{\Delta X \rightarrow 0} \frac{\Delta Q}{\Delta X}$.

In the solar wind $\Delta Q$ should be self-similar - that is, independent not only of $\Delta X$ but also of time. The result is that $\frac{\Delta Q}{\Delta X}$ exhibits a dependence on $\Delta X$. To remove the dependence the derivative is estimated at a number of spacecraft separations and the results are interpolated to back to $\Delta X=0$. To remove the fluctuations in $\Delta Q$ requires a statistically significant number of estimates of the derivative to be made at each spacecraft separation. How this effects the estimation of $\lambda_{\mathrm{T}}$ and how it is corrected is discussed explicitly in Sect. 3.4.

In addition to the two limitations listed above, we also require during intervals used that the spacecraft be in a nearregular tetrahedral configuration. This is judged from the value of the tetrahedron geometry parameter (QGM) (Robert et al., 1998), which varies between 1 and 3, with 3 indicating that the spacecraft are in a nearly regular tetrahedral geometry and 1 indicating the spacecraft are approximately in a line. There is no a priori QGM value above which the spacecraft can be considered to be in a near tetrahedron, but Fig. 3 suggests that a QGM above 2.7 should be adequate. The figure contains two panels. The upper panel is the estimated curl of the magnetic field when each component of the field has been artificially set to a constant value of $5000 \mathrm{nT}$ on each spacecraft. Ideally the curl should be zero everywhere. The lower panel shows the spacecraft QGM for the configuration found on 26 February 2005 between 10:00 and 21:00 UT. This configuration, which had a QGM varying from 3 to about 2.1, was used to compute the curl. Jitter in the determined spacecraft positions and hence in the relative spacings is responsible for the hash seen in the curl. The overall increase in magnitude beginning about 15:30 UT, however, is due to changes in the $(x, y, z)$ spacecraft separation that begins to move the spacecraft away from its original nearly regular tetrahedral configuration. The overall spacecraft positions were continuously adjusted across the $11 \mathrm{~h}$ interval (without affecting the configuration) to keep a constant average separation. This removes any functional dependence on $\lambda_{\mathrm{T}}$ from the magnitude of the separation itself. As seen in the top plot the estimated $\lambda_{\mathrm{T}}$ is reasonably constant up until about hour 16, where the upward trend begins. This upward trend occurs at a QGM value of roughly 2.7.

\subsection{Estimating $\lambda_{T}$}

Estimates of $\lambda_{\mathrm{T}}$ from either magnetic field or electron fluid velocity data are constructed directly from Eq. (2) and require only the formation of the two ensemble averages consisting of the square of the curl and the square of the magnitude of the fluctuating component of the parameter in question. The method used to estimate the curl has been detailed in Sect. 3.3. The magnitude is obtained through Eq. (4) at the position of the centroid (average of the vertices) of the volume defined by the spacecraft.

The intervals of time over which the ensemble averages are formed must meet several conditions, some of which have already been discussed above and are restated here. The spacecraft must be in a reasonably regular tetrahedral formation throughout the interval. We ensure this by requiring that the average QGM be greater than 2.75 over the time period. (The condition is sometimes relaxed to 2.65 when the electron fluid velocity is used to estimate $\lambda_{\mathrm{T}}$ for the simple reason that periods of burst mode telemetry do not always occur when the spacecraft are in an optimal tetrahedral configuration.) The time periods during which the magnetic-field-based $\lambda_{\mathrm{T}}$ are computed generally have an average QGM above 2.95 except for times in 2009, where the positioning of $\mathrm{C} 3$ and $\mathrm{C} 4$ did not allow for QGM values much above 2.8. Also, during orbits for which the spacecraft had two periods of highquality tetrahedral configurations in the solar wind, one generally has a average QGM below 2.85 but above 2.75 . There are no data in the study from 2007 to 2008 because in that time frame $\mathrm{C} 3$ and $\mathrm{C} 4$ were very close, while the remaining spacecraft were separated by $\sim 10000 \mathrm{~km}$. The QGM in those years did not increase much above 2 .

In addition, the time intervals must be long enough that the ensemble averages contain sufficient data to be statistically 
significant. With the 5 VPS magnetic field data we generally use $70 \mathrm{~min}$ intervals, which contain about 21000 individual measurements. The slower cadence of the electron velocity moment $(\sim 4 \mathrm{~s})$ means that to obtain statistically significant ensemble averages, longer time intervals are required. As with the QGM, where we must be satisfied with lower values than we would like to see being limited by the duration of the burst mode, which generally ranges from 1.5 to $3 \mathrm{~h}$ in duration (1350 to 2700 measurements). Burst mode intervals shorter than $1.5 \mathrm{~h}$ were not included.

A last requirement in the selection of time intervals is that the measurements at each spacecraft be independent of one another. This means that we need to ensure that features in the solar wind do not propagate across multiple spacecraft within a measurement time frame. Another way of saying this is that

$$
\frac{D}{V_{\mathrm{sw}}}>t_{\mathrm{M}}
$$

where $D$ is the average spacecraft separation (see Eq. 9 below), $V_{\mathrm{sw}}$ is the solar wind speed, and $t_{\mathrm{M}}$ is the time resolution of the measurement. This places limits on the spacecraft separation that can be used for the different data sets. For the $0.2 \mathrm{~s}$ magnetic field data the minimum average separation for an $800 \mathrm{~km} \mathrm{~s}^{-1}$ solar wind speed should be on the order of $200 \mathrm{~km}$, while for the $4 \mathrm{~s}$ fluid velocity the same minimum separation would be about $3500 \mathrm{~km}$. The latter limits the use of the electron fluid velocity to the years 2003 and 2006.

The sum requirements have a significant impact on the number of time intervals available for analysis when using electron velocity data, but minimal impact on the number of intervals available when using magnetic field data. As such, most of the analysis was carried out using the magnetic field data.

As mentioned in Sect. 3.2, the use of different cutoff frequencies to remove the mean field is equivalent to specifying the scale length over which $\lambda_{\mathrm{T}}$ is estimated. This inherently changes the estimated $\lambda_{\mathrm{T}}$ as more power (numerator in Eq. 2) is included in the estimation with longer scale lengths. Figure 4 shows $\lambda_{\mathrm{T}}$ estimated from magnetic field data within a 20 min window as a function of the cutoff filter frequency (equivalent scale length is shown on the upper $x$ axis). This shows the falloff in $\lambda_{\mathrm{T}}$ with increasing cutoff frequency (decreasing scale length). The red line is a fit to the data, and it is easy to see how one could, in principle, extend the fit to estimate $\lambda_{\mathrm{T}}$ over the full correlation length. However, because $\lambda_{\mathrm{T}}$ still contains a functional dependence on the spacecraft separation at this point, the projected value would just be an intermediate result that would still need to be corrected for the spacecraft separation.

The effect of spacecraft separation on the estimated $\lambda_{\mathrm{T}}$ is shown in Fig. 5. The first eight plots in the figure show the estimated $\lambda_{\mathrm{T}}$ from the magnetic field data as a function of average spacecraft separation. Each plot contains estimates from the same 138 seventy-five-minute intervals between
Cluster MF Taylor Microscale Estimates

Based on 0.02s Magnetic Field Data

2003 051 06:15:00.000 TO 2003051 06:45:00.000

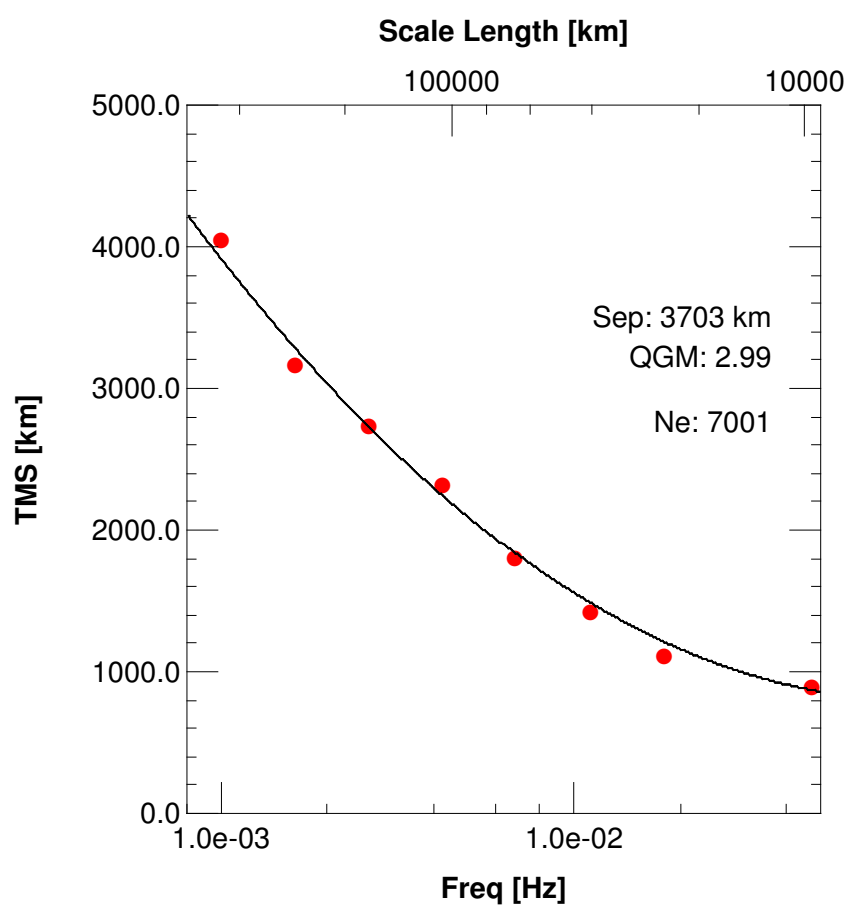

Fig. 4. $\lambda_{\mathrm{T}}$ from magnetic field data as a function of the cutoff filter frequency and scale length (upper axis) showing the increase in the estimated $\lambda_{\mathrm{T}}$ with increasing scale length and decreasing cutoff frequency.

2001 and 2009 derived from the different cutoff filters. The spacecraft separation is the average separation of all spacecraft determined from

$D=\frac{1}{6} \sum_{j=0}^{j=2} \sum_{i=j}^{i=3} \sqrt{\left(x_{i}-x_{j}\right)^{2}+\left(y_{i}-y_{j}\right)^{2}+\left(z_{i}-z_{j}\right)^{2}}$,

where $i$ and $j$ represent pairs of the Cluster spacecraft. The cutoff filters represent different scale lengths over which $\lambda_{\mathrm{T}}$ was estimated. The relationship between cutoff filter and scale length is shown in the lower right-hand plot in Fig. 5.

The red lines in the $\lambda_{\mathrm{T}}$ plots are linear least-squared fits of the form $\lambda_{\mathrm{T}}=a r+b$ to the data ( $r$ is the average spacecraft separation and $a$ and $b$ are the fit coefficients). These appear to be good representational fits to the data and consistent with our understanding of how the artificial dependence on the spacecraft separation introduced in the spatial derivatives is expected to behave. They essentially map out the average $\lambda_{\mathrm{T}}$ value at each average tetrahedral separation distance. $\lambda_{\mathrm{T}}$ at $0 \mathrm{~km}$ separation $\left(\lambda_{\mathrm{T}}(0)\right)$ is given in each plot as determined from the fit. This is the estimated $\lambda_{\mathrm{T}}$ for the scale length represented by the filter. The organization of $\lambda_{T}$ values along vertical lines in the plots is due to the fact that 


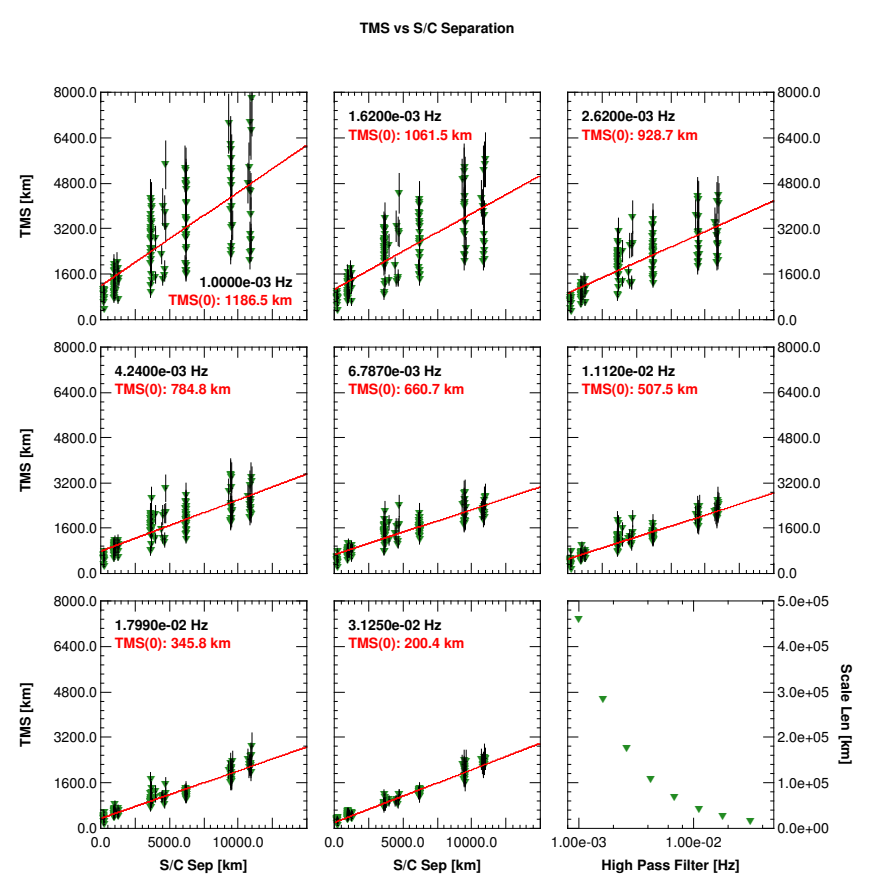

Fig. 5. Functional dependence of the estimated $\lambda_{\mathrm{T}}$ values from magnetic field data on the spacecraft separation for all eight cutoff filter frequencies. The cutoff filter frequency as well as $\lambda_{\mathrm{T}}$ at zero spacecraft separation are given in each plot. The latter was computed directly from the fits. The bottom right-hand plot shows the relationship between $\lambda_{\mathrm{T}}(0)$ and the cutoff filter frequencies.

the spacecraft enter optimal tetrahedron formations at about the same separations in each orbit. Multiple lines are the result of either changes in the overall spacecraft separations or times when the spacecraft pass through two periods of tetrahedral formation in the solar wind per orbit, each occurring at different average separations.

It should be recognized that the functional dependency of TMS on the spacecraft separation as seen in Fig. 5 is generated on an event-by-event basis. The spacecraft separation (as well as configuration), however, changes continually, and therefore the same dependency is introduced into the individual ensemble averages. This change in separation introduces a small spread in the ensemble averages that translates into an equivalent spread in the estimated TMS. The spread becomes more pronounced as the time interval increases. We handle this by computing the average spacecraft separation within the time interval and then defining a scaling factor $\epsilon$ at each measurement as $\langle D\rangle / D$. This scaling factor is then used to rescale the spacecraft separations, thus allowing us to use a constant separation for all calculations within the time window without changing the QGM, effectively removing the spacecraft separation dependency from the ensemble averages.
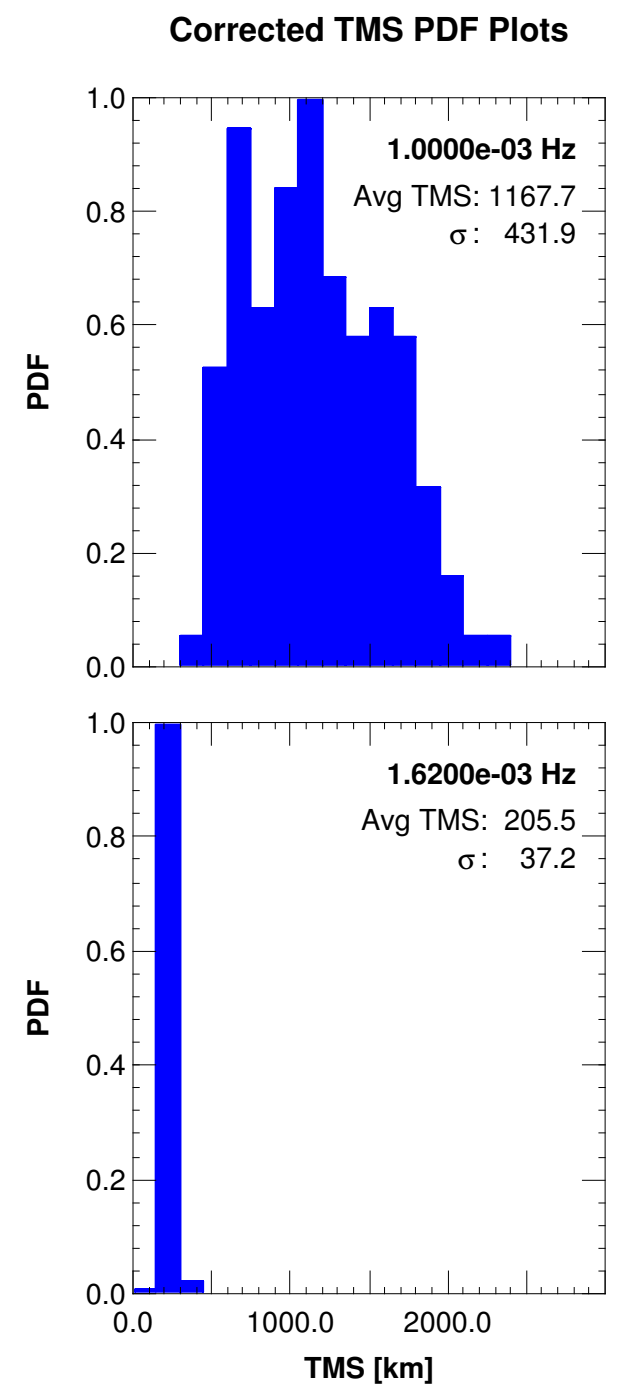

Fig. 6. Probability distribution function of the corrected $\lambda_{\mathrm{T}}$ values at two cutoff frequencies. Both the average $\lambda_{\mathrm{T}}$ and the standard deviation are given in the plot boxes. The bin size is $150 \mathrm{~km}$ in both plots.

One feature that is very noticeable in Fig. 5 is the increase in the spread of the estimated $\lambda_{\mathrm{T}}$ with decreasing cutoff filter frequency (or equivalently increasing scale length). Increasing the scale length over which $\lambda_{\mathrm{T}}$ is estimated increases the total power (numerator in Eq. 2) included in the $\lambda_{\mathrm{T}}$ estimate. This in turn increases the variation in values obtained from different evaluations.

The functional dependence of $\lambda_{\mathrm{T}}$ on the spacecraft separation in any of the plots in Fig. 5 can be removed by using the linear fits to map the individual points back to $\lambda_{\mathrm{T}}(0)$. Doing this allows us to construct a probability distribution function (PDF) of the corrected $\lambda_{\mathrm{T}}$, as shown in Fig. 6 for two sets of $\lambda_{\mathrm{T}}$ values estimated from the 0.0162 and $0.001 \mathrm{~Hz}$ filter cutoffs. This example illustrates both the increase in average 
TMS and $\sigma$ Plots
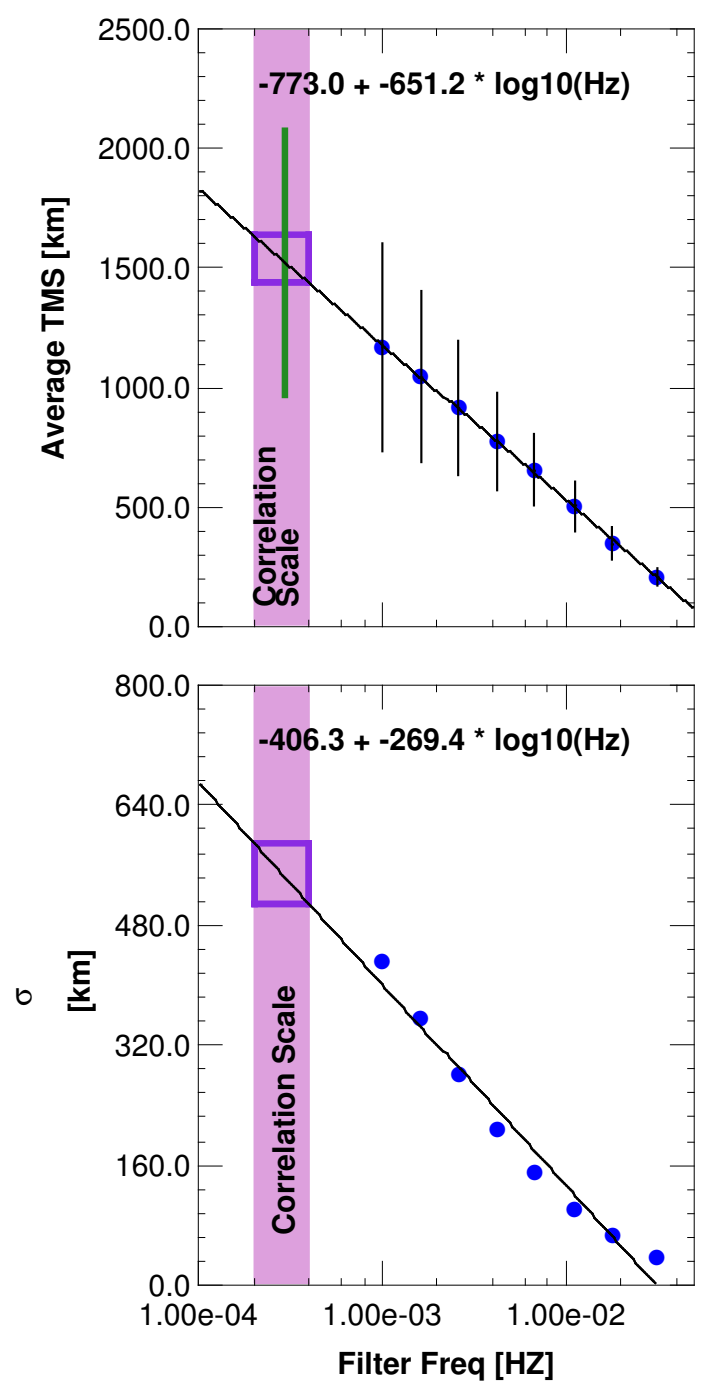

Fig. 7. $\lambda_{\mathrm{T}}$ and corresponding standard deviation as a function of filter frequency (or equivalently the scale length). The linear relationship is used to project $\lambda_{\mathrm{T}}$ back to the correlation scale (purple band).

$\lambda_{\mathrm{T}}$ and the corresponding increase in the standard deviation with increasing scale length.

As noted in the introduction and seen in Fig. 5, the method used to derive $\lambda_{\mathrm{T}}$ above does not cover the full correlation scale length. Using spectra of the magnetic field turbulence within the time frame of the intervals analyzed, we estimate the correlation length to be on the order of $2 \times 10^{6} \mathrm{~km}$ (obtained from selected time intervals that were greater than 4 times the correlation length), while Fig. 7 shows that we only cover a scale length of about $5 \times 10^{5} \mathrm{~km}$. We can, however, estimate $\lambda_{\mathrm{T}}$ over a correlation length by projecting $\lambda_{\mathrm{T}}$ estimated from the smaller scale lengths as shown in Fig. 5 out to the correlative scale. The upper plot shows the average
$\lambda_{\mathrm{T}}$ values obtained from the corrected $\lambda_{\mathrm{T}}$ at each cutoff frequency and the lower plot the corresponding standard deviations. Both are fit to a linear function with the fit coefficients shown. The error bars on $\lambda_{\mathrm{T}}$ are from the standard deviations. The shaded region in both plots shows the location of the correlation scale (the width representing the uncertainty), and the boxed region within shows the range of $\lambda_{\mathrm{T}}$ and standard deviations expected from projecting both values to the correlation scale. This translates to a value of $1538 \pm 550 \mathrm{~km}$ for $\lambda_{\mathrm{T}}$. The average ion inertial length over the time periods during which $\lambda_{\mathrm{T}}$ was computed was found to be $\sim 100 \mathrm{~km}$. The ion inertial length is a reasonable estimate for the scale length associated with the dissipation region. This places $\lambda_{\mathrm{T}}$ well above the dissipation scale length.

The solar wind $\lambda_{\mathrm{T}}$ computed through this formulation is about $850 \mathrm{~km}$ smaller than that reported by Weygand et al. (2007) $(2400 \pm 100 \mathrm{~km})$. The extreme limits of the two values are only separated by about $200 \mathrm{~km}$. Some of the difference may be due to a different mix of foreshock/solar wind data in the two analysis. It is not known if $\lambda_{\mathrm{T}}$ is the same is both regions. Considering, however, the differences in the methods and approximations used, this probably is not an unreasonable difference.

\section{Comparisons between magnetic field and velocity estimates of $\lambda_{T}$}

One of the goals of this study was to compare $\lambda_{\mathrm{T}}$ estimated from the magnetic data to that estimated from the electron fluid velocity. While we have demonstrated the feasibility of doing this, the small number of time periods (six in all) during which the electron data met all of the criteria outlined in Sect. 3.4 does not allow for the type of statistical analysis required to determine the functional relationship of $\lambda_{\mathrm{T}}$ with distance, which is necessary to determine $\lambda_{\mathrm{T}}$ over a correlation length. We can, however, compare results on an interval-by-interval basis. In a case-by-case comparison, $\lambda_{\mathrm{T}}$ is estimated from both electron and magnetic field data over the same spacecraft separation and QGM values. $\lambda_{\mathrm{T}}$ estimated from both the magnetic field and electron fluid velocity for two of the six intervals looked at are shown in Fig. 8. These show $\lambda_{\mathrm{T}}$ as a function of the cutoff filter frequency (the same format as used in Fig. 4). The magnetic field data in these examples has been averaged to the spacecraft spin period $(\sim 4 \mathrm{~s})$ to match the time resolution of the velocity measurements. In both plots the magnetic-field-based $\lambda_{\mathrm{T}}$ becomes more and more separated from the velocity-based estimates with increasing scale length. This behavior was observed in five of the six cases we examined. In the sixth case (not shown) the two curves nearly overlaid one another and neither exhibited an upward trend with increasing scale length.

As seen in the plots the two $\lambda_{\mathrm{T}}$ estimates diverge with increasing scale length but are roughly equivalent at smaller 


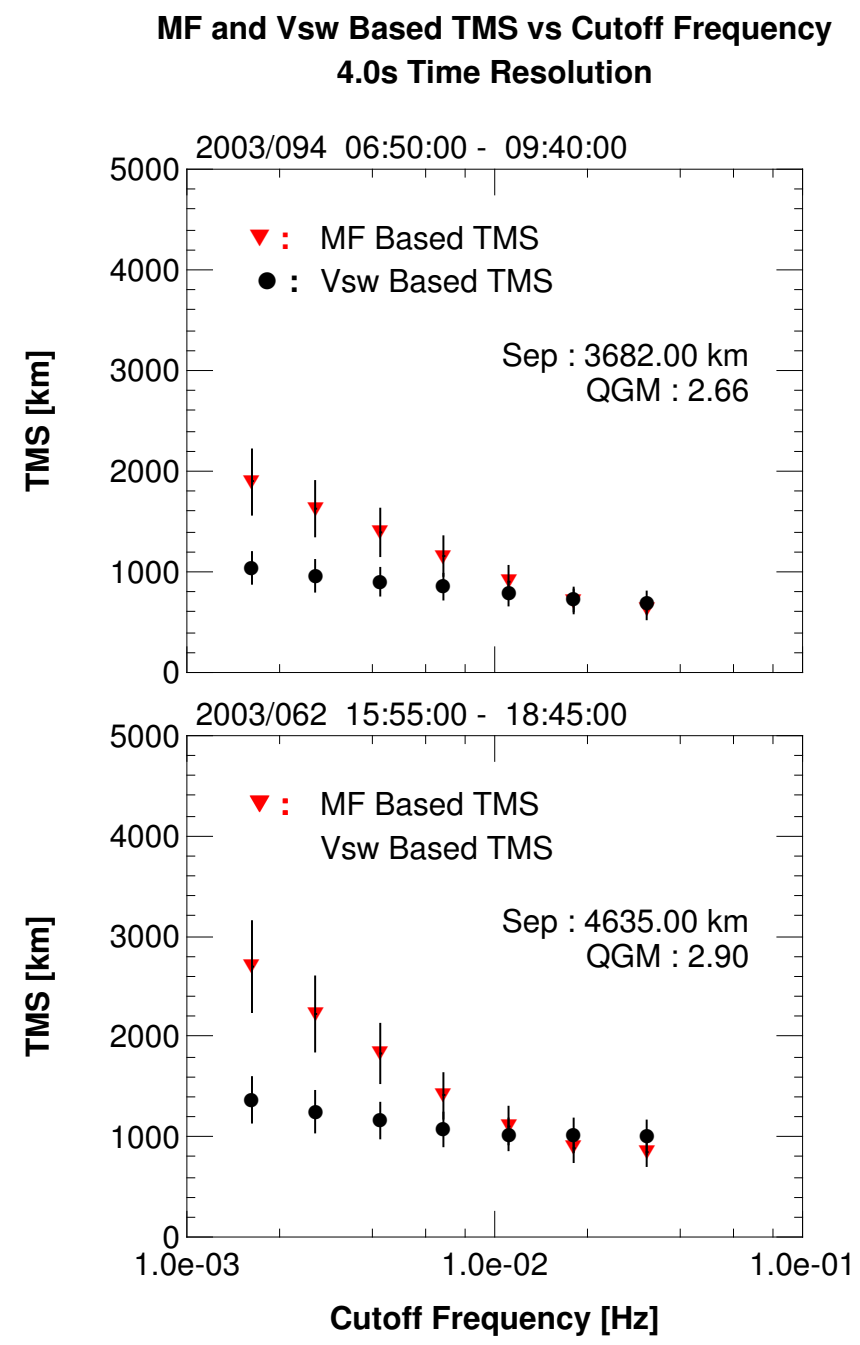

Fig. 8. Plots of the magnetic field and velocity-based $\lambda_{\mathrm{T}}$ as a function of cutoff frequency for two time intervals. The magnetic-fieldbased values increasingly separate from the velocity-based values with increasing scale length (decreasing cutoff filter).

scale length. This was seen in all cases looked at. As the filter frequency is increased, more and more of the energy that resides in the fluctuations is removed. Over the entire correlative range the energy in the magnetic fluctuations is much larger than in the velocity fluctuations (Salem et al., 2009; Chen et al., 2013). At some frequency, however, you get to a point where the magnetic field and velocity power falls roughly into the same range and the curves overlay.

Why the velocity-based $\lambda_{\mathrm{T}}$ appears to be less than the comparable magnetic-field-based values is not clear, however, from the few time intervals available. In collisionless low-density plasma, which the solar wind is, the basic turbulence scale lengths are controlled by the plasma and field characteristics that near $\lambda_{\mathrm{T}}$ are both dynamic and complex (see Matthaeus et al., 2008). It is possible that the magnetic field and solar wind velocity partially decouple over the scale

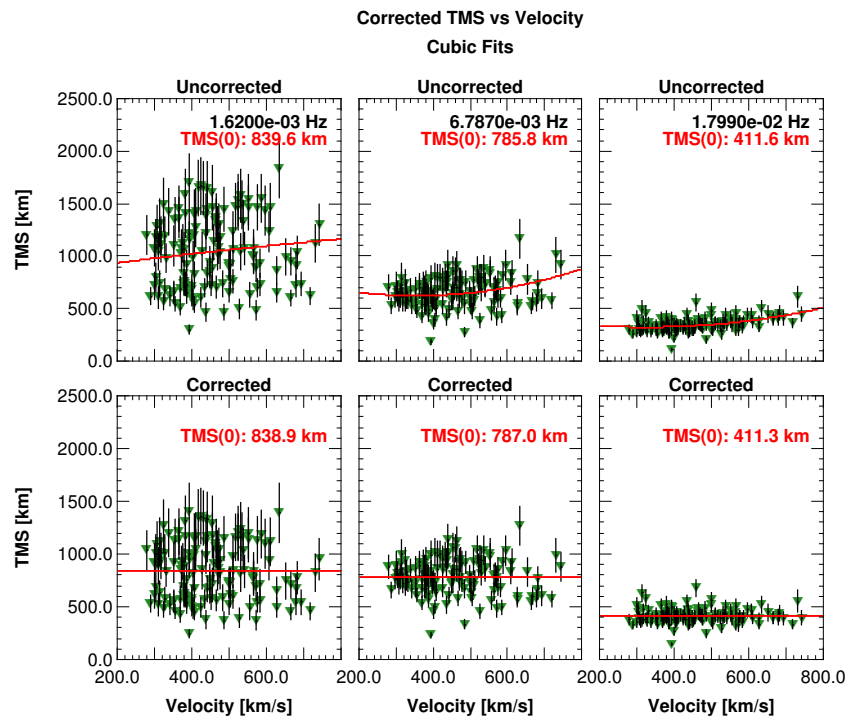

Fig. 9. Plots of $\lambda_{\mathrm{T}}$ vs. the solar wind speed for various cutoff frequencies. The plots show a very small dependence of $\lambda_{\mathrm{T}}$ on velocity.

lengths of the analysis, which allows for a separation in the physics that pushes the velocity-based $\lambda_{\mathrm{T}}$ to smaller values. The results suggest that if the dissipation scale for the two parameters is identical, the velocity-based $\lambda_{\mathrm{T}}$ lies closer to it so that the dissipation of magnetic field energy begins at slightly larger scales compared to the velocity. However, it might also be indicative of a difference in the location of the dissipation scale between the two parameters.

\section{Possible dependence of $\lambda_{T}$ on solar wind speed}

Following the lead of Weygand et al. (2011) we have looked at the possibility of a dependence of $\lambda_{\mathrm{T}}$ on the solar wind speed. Once the spacecraft separation dependence is removed we can produce plots of $\lambda_{\mathrm{T}}$ as a function of the solar wind speed. Figure 9 shows plots of $\lambda_{\mathrm{T}}$ as a function of solar wind speed at three different cutoff filter frequencies. The top row of the plot shows the distance-corrected $\lambda_{\mathrm{T}}$ that have been fit to a cubic function (red curve). The cubic fit, at least at the larger cutoff filters, is a better representation of the data than is a linear fit because of the slight upward curvature in the data at higher solar wind speeds. The bottom row of plots show the speed-corrected $\lambda_{\mathrm{T}}$ (solar wind speed dependence removed). The red curves in these plots are linear fits, which give some indication as to how well the cubic fit correction worked.

The speed dependency, if real, is small and may represent a manifestation of the velocity dependence in $\lambda_{\mathrm{T}}$ anisotropy reported by Weygand et al. (2011). The dependency is much easier to visualize at the higher cutoff frequencies (two righthand columns of plots) where the scatter in $\lambda_{T}$ is small. As the cutoff frequency decreases the scatter in $\lambda_{\mathrm{T}}$ masks any 
organization with speed that may exist in the data (see the left-hand column of plots). It is possible that the trend in the data at the higher cutoff filters is statistical, being due to the lack of samples at the higher speeds. It should be emphasized that $\lambda_{\mathrm{T}}$ presented here are estimates constructed from a statistical analysis of many individual results, and as such significant variability should always be expected.

\section{Conclusions}

In this paper we have estimated $\lambda_{\mathrm{T}}$ by using data from the four Cluster spacecraft to form spatial derivatives that can be used in the relationship given in Eq. (2). This approach makes no assumptions about the isotropy of the turbulence (but does assume that the variation of the magnetic and velocity fields across the tetrahedron formed by the four spacecraft is linear). In applying the methodology we have obtained a $\lambda_{\mathrm{T}}$ in the solar wind based on the magnetic field of about $1538 \pm 550 \mathrm{~km}$, which is about $850 \mathrm{~km}$ less than that obtained by Weygand et al. (2007). The values are much closer when including the errors on both estimates but still do not overlap. The reason for that difference is not altogether clear. For the small number of cases where the relative $\lambda_{\mathrm{T}}$ can be computed from both the magnetic field and the electron fluid velocity, values derived from the electron fluid velocity appear to produce lower values. The difference increases with increasing scale length (see Fig. 6 and remember that the cutoff filter frequency is equivalent to scale length). Perhaps this should not be surprising since the dissipation mechanisms associated with magnetic field and electron fluid velocity near the Kolmogorov scale are not necessarily the same. It is only recently that the turbulence spectra for the solar wind velocity has been extended to the ion kinetic scale (Šafránková et al., 2013). The velocity was obtained at a cadence of $31 \mathrm{~ms}$ from a three point ion distribution function. The results show an average break in the velocity spectra of $0.38 \mathrm{~Hz}$, which, using a solar wind speed of $477 \mathrm{~km} \mathrm{~s}^{-1}$ (the average of the range of the solar wind speeds in their study), gives a scale length of about $1250 \mathrm{~km}$. It is unfortunate that a comparable set of simultaneous spectra for the magnetic field data was not reported as this would have allowed direct comparisons between the break points to be made. The value is, however, within the range of reported break points for magnetic field spectra reported in Perri et al. (2010) and Bourouaine et al. (2012).

It is, however, instructive to compare the magnetic field and electron fluid velocity power spectra over the common frequency range covered by each. This is shown in Fig. 10 using the time period covered in the lower plot in Fig. 8. (We have also included a spectrum generated from the velocity estimated from the CIS ion experiment on C1.) The difference in time resolution between the two data sets $(0.02 \mathrm{~s}$ for the magnetic field data and $4 \mathrm{~s}$ for the velocity data) accounts for the difference in frequency range (recall that the mag-

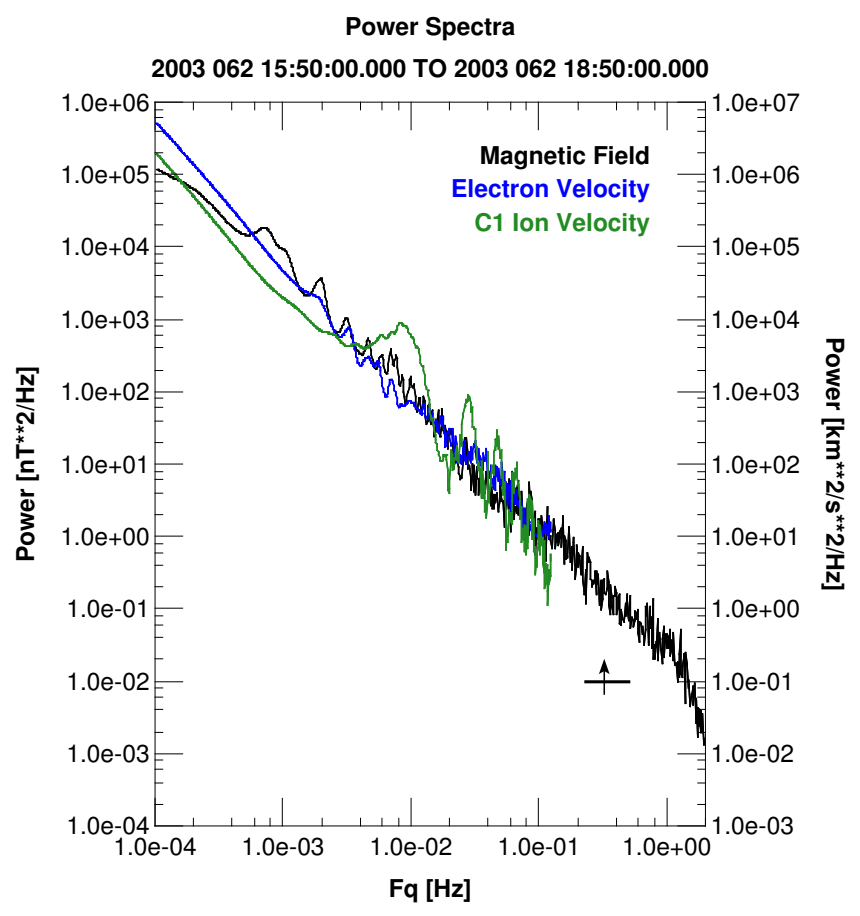

Fig. 10. Example spectra of the magnetic field (black - left-hand axis) and the electron and ion fluid velocity (blue and green, respectively - right-hand axis) for the same time period as shown in the lower plot in Fig. 8. The time resolution of the magnetic field and velocity data was 0.2 and $4 \mathrm{~s}$, respectively. The electron velocity data have had high-frequency noise above $0.06 \mathrm{~Hz}$ removed. This tends to flatten the spectra. The spectral indices of the magnetic and velocity spectra are about -1.7 . The arrow shows the position of the $\lambda_{\mathrm{T}}$ scale estimated from this study.

netic field data used in the $\lambda_{\mathrm{T}}$ plots in Fig. 8 were averaged to $4 \mathrm{~s}$ to match the cadence of the velocity measurements). The magnetic field spectrum extends high enough in frequency to show the Kolmogorov steepening beginning at about $1 \mathrm{~Hz}$, while the velocity spectra can be carried out only to $0.125 \mathrm{~Hz}$ (Nyquist frequency). It should also be noted that the electron velocity data have been filtered to remove most of the highfrequency noise above $0.06 \mathrm{~Hz}$, which if not removed causes the spectra to flatten at the higher frequencies. The arrow in the figure denotes the location of the $\lambda_{\mathrm{T}}$ scale estimated in this study, while the horizontal line indicates the error range. The scale is slightly above the spectral break at $1 \mathrm{~Hz}$.

All three spectra have near-identical spectral indices of about -1.7 over their common frequency range, close to the Kolmogorov spectral index of $-5 / 3$. The presence of backscattered electrons within the time frame the spectra were constructed indicates that the spacecraft were within the foreshock (Larson et al., 1996), which may account for the multiple distinct peaks in the ion velocity spectra. The near-Kolmogorov spectra in the velocity spectra are not in agreement with the -1.5 spectral index reported in a number of papers (e.g., Podesta et al., 2007); however it should 
be noted that the data used in those papers were acquired in the free-streaming solar wind, whereas these measurements come from the foreshock. This also might account for the fact that the location of the beginning of the spectral steepening at the start of the ion dissipation region $(1 \mathrm{~Hz})$ is lower than the nominal $0.5 \mathrm{~Hz}$ seen in the solar wind (v., e.g., Sahraoui et al. 2009). The common spectral slopes in the spectra suggests that the differences in the observed values of $\lambda_{\mathrm{T}}$ generated from the magnetic field and velocity data are due to differences in the turbulence spectra within the intervals used in the analysis.

As noted in the introduction, while the formalism used to define $\lambda_{\mathrm{T}}$ is based on a hydrodynamic turbulence model, near the dissipation scale the solar wind does not behave as a classical fluid (Matthaeus et al., 2008). The differences in behavior are probably the reason that the location of the estimated $\lambda_{\mathrm{T}}$ within the inertial range, as shown in Fig. 10, does not appear to follow the $2 / 3-1 / 3$ separation distance from the inertial and dissipation scales, as would be expected in a fully fluid medium. This may be looked at as a problem in defining the location of the dissipation scale. In keeping with fluid terminology, the termination of the inertial range at spectral break just above the Taylor microscale is often defined as the dissipation scale. The kinetic effects that terminate the inertial range, however, may not be fully dissipative in nature but instead dispersive. The spectral break at the end of the inertial scale is generally associated with the local ion scale, and there is a similar break at higher frequencies (not seen in Fig. 10 due to the temporal resolution of the data) associated with the electron scale (see Sahraoui et al., 2009). Where one defines the dissipation scale - at the ion scale, the electron scale, or some average of the two - is an open question.

Because the formulation used to estimate $\lambda_{\mathrm{T}}$ in this paper uses the vector representation of the curl, it may be possible to extend the technique to estimate $\lambda_{\mathrm{T}}$ along arbitrary directions with respect to the magnetic field. This would allow for investigation of possible anisotropies in $\lambda_{\mathrm{T}}$.

Acknowledgements. The authors would like to acknowledge the work and role the Cluster Active Archive (CAA) and thank the CIS, EFW, WHISPER, and FGM teams for providing the data used in this study. We would also like to acknowledge the PEACE team for access to the high-resolution electron data. C. Gurgiolo would like to acknowledge support from NASA grant NNX10AQ46G. W. H. Matthaeus would like to acknowledge support from the NASA Guest Investigator Program grant NNX09AG31G, the NASA Heliophysics Theory Program grant NNX11AJ44G, and the NSF Shine Program grant AGS-1156094. M. L. Goldstein and A. Viñas acknowledge support of the NASA Cluster Mission.

Topical Editor R. Nakamura thanks L. Sorriso-Valvo and two anonymous referees for their help in evaluating this paper.

\section{References}

Balogh, A., Dunlop, M. W., Cowley, S. W. H., Southwood, D. J., Thomlinson, J. G., Glassmeier, K. H., Musmann, G., Lühr, H., Buchert, S., Acuña, M. H., Fairfield, D. H., Slavin, J. A., Riedler, W., Schwingenschuh, K., and Kivelson, M. G.: The CLUSTER magnetic field investigation, Space Sci. Rev., 79, 65-91, 1997.

Barnes, A.: Hydromagnetic waves and turbulence in the solar wind, in: Solar System Plasma Physics, Vol. 1, edited by: Parker, E. N., Kennel, C. F., and Lanzerott, L. J., p. 249, Cambridge: Cambridge Univ. Press, Amsterdam: North-Holland, 1979.

Batchelor, G. K.: Basic space plasma physics, University of Cambridge, Melbourne, Australia, 1953.

Belmabrouk, H. and Michard, M.: Taylor length scale measurement by laser Doppler velocimetry, Exp. Fluids, 25, 69-76, 1998.

Borovsky, J. E.: A model for the MHD turbulence in the Earth's plasma sheet: Building computer simulations, in: Multiscale processes in the Earth's magnetosphere from Interball to Cluster, edited by: Sauvaud, J. A. and Němeček, Z., 217-249, Kluwer Academic Publishers, The Netherlands, 2004.

Bourouaine, S., Alexandrova, O., Marsch, E., and Maksimovic, M.: On spectral breaks in the power spectra of magnetic fluctuations in the fast solar wind between 0.3 and 0.9 AU, Astrophys. J., 749, 749-755, doi:10.1088/0004-637X/749/2/102, 2012.

Chen, C. H. K., Bale, S. D., Salem, C. S., and Maruca, B. A.: Residual Energy Spectrum of Solar Wind Turbulence, Astrophys. J., 770, 125, doi:10.1088/0004-637X/770/2/125, 2013.

Gurgiolo, C., Goldstein, M. L., Viñas, A. F., and Fazakerley, A. N.: First measurements of electron vorticity in the foreshock and solar wind, Ann. Geophys., 28, 2187-2200, doi:10.5194/angeo-282187-2010, 2010.

Gurgiolo, C., Goldstein, M. L., A. F. Viñas, W. H. M., and Fazakerley, A. N.: Observations of electron vorticity in the inner plasma sheet, Ann. Geophys., 29, 1517-1527, doi:10.5194/angeo-291517-2011, 2011.

Holzer, T. E., Leer, E., and Zhao, X.-P.: Viscosity in the solar wind, J. Geophys. Res., 91, 4126-4132, doi:10.1029/JA091iA04p04126, 1986.

Johnstone, A. D., Alsop, C., Gurge, S., Carter, P. J., Coates, A. J., Coker, A. J., Fazakerley, A. N., Grande, M., Gowen, R. A., Gurgiolo, C., Hancock, B. K., Narheim, B., Preece, A., Sheather, P. H., Winningham, J. D., and Woodcliffe, R. D.: PEACE: A plasma electron and current experiment, Space Sci. Rev., 79, 351-398, 1997.

Kailasnath, P. and Sreenivasan, K. R.: Zero crossings of velocity fluctuations in turbulent boundary layers, Phys.Fluids A, 11, 2879-2885, 1993.

Larson, D. E., Lin, R. P., McFadden, J. P., Ergun, R. E., Carlson, C. W., Anderson, K. A., Phan, T. D., McCarthy, M. P., Parks, G. K., Réme, H., Bosqued, J. M., d'Uston, C., Sanderson, T. R., Wenzel, K. P., and Lepping, R. P.: Probing the Earth's bow shock with upstream electrons, Geophys. Res. Lett., 23, 2203-2206, doi:10.1029/96GL02382, 1996.

Leamon, R. J., Matthaeus, W. H., Smith, C. W., and Wong, H. K.: Contribution of cyclotron-resonant damping to kinetic dissipation of interplanetary turbulence, Astrophys. J., 507, L181-L185, 1998. 
Matthaeus, W. H., Dasso, S., Weygand, J. M., Milano, L. J., Smith, C. W., and Kivelson, M. J.: Spatial correlation of the solar wind turbulence from two point measurements, Phys. Rev. Lett., 95, 231101-231104, doi:10.1103/PhysRevLett.95.231101, 2005.

Matthaeus, W. H., Weygand, J. M., Chuychai, P., Dasso, S., Smith, C. W., and Kivelson, M. J.: Interplanetary magnetic Taylor microscale and implications for plasma dissipation, Astrophys. J., 678, L141-L144, 2008.

Perri, S., Carbone, V., and Veltri, P.: Where does fluid-like turbulence break down in the solar wind?, Astrophys. J.(Lett.), 725, L52-L55, doi:10.1088/2041-8205/725/1/L52, 2010.

Podesta, J. J., Roberts, D. A., and Goldstein, M. L.: Spectral exponents of kinetic and magnetic energy spectra in solar wind turbulence, Astrophys. J., 664, 543-548, 2007.

Press, W. H., Teukolsky, S. A., Vetterling, W. T., and Flannery, B. P.: Numerical recipes in C: The art of scientific computing, Cambridge University Press, The Edinburgh Building, Cambridge, CB2 2RU, UK, 1999.

Robert, P., Roux, A., Harvey, C. C., Dunlop, M. W., Daly, P. W., and Glassmeier, K.-H.: Tetrahedron Geometry Factors, in: Analysis methods for multi-spacecraft data, edited by: Paschmann, G. and Daly, P. W., 323-348, ESA Publications Division, Keplerlaan 1, 2200 AG Noordwijk, The Netherlands, 1998.

Šafránková, J., Nêmeĉek, Z., Pr̂ech, L., and Zastenker, G.: Ion Kinetic Scale in the Solar Wind Observed, Phys. Rev. Lett., 110, 024004, doi:10.1103/PhysRevLett.110.025004, 2013.

Sahraoui, F., Goldstein, M. L., Robert, P., and Khotyaintsev, Y. V.: Evidence of a cascade and dissipation of solar wind turbulence at electron scales, Phys. Rev. Lett., 102, 231102, doi:10.1103/PhysRevLett.102.231102, 2009.

Salem, C., Mangeney, A., Bale, S. D., and Veltri, P.: Solar wind magnetohydrodynamics turbulence: Anomalous scaling and role of intermittency, Astrophys. J., 702, 537-553, doi:10.1088/0004637X/702/1/537, 2009.

Salem, C. S., Howes, G. G., D.Sundkvist, Bale, S. D., Chaston, C. C., Chen, C. H. K., and Mozer, F. S.: Identification of kinetic Alfvén wave turbulence in the solar wind, Astrophys. J., 745, L9-L13, doi:10.1088/2041-8205/745/1/L9, 2012.
Segalini, A., Örlü, R., Schlatter, P., Rüedi, J.-D., and Talamelli, A.: A method to estimate turbulence intensity and transverse Taylor microscale in turbulent flows from spatially averaged hot-wire data, Exp. Fluids, 51, 693-700, doi:10.1007/s00348-011-1088$0,2011$.

Taylor, G. I.: Statistical theory of turbulence, Proc. R. Soc. Lond. A, 151, 421-444, doi:doi: 10.1098/rspa.1935.0158, 1935.

Taylor, G. I.: The Spectrum of Turbulence, in: Proceedings of the Royal Society of London. Series A, pp. 476-490, 1938.

Tu, C. Y. and Marsch, E.: MHD structures, waves and turbulence in the solar wind: Observations and theories, Space Sci. Rev., 73, $1-210,1995$.

Verma, M. K.: Nonclassical viscosity and resistivity of the solar wind plasma, J. Geophys. Res., 101, 27543-27548, doi:10.1029/96JA02324, 1996.

Weygand, J. M., Matthaeus, W. H., Dasso, S., Kivelson, M. G., and Walker, R. J.: Taylor scale and effective magnetic Reynolds number determination from plasma sheet and solar wind magnetic field fluctuations, J. Geophys. Res., 112, A10201, doi:10.1029/2007JA012486, 2007.

Weygand, J. M., Matthaeus, W. H., Dasso, S., Kivelson, M. G., Kistler, L. M., and Mouikis, C.: Anisotropy of the Taylor scale and the correlation scale in plasma sheet and solar wind magnetic field fluctuations, J. Geophys. Res., 114, A07213, doi:10.1029/2008JA013766, 2009.

Weygand, J. M., Matthaeus, W. H., El-Alaoui, M., Dasso, S., and Kivelson, M. G.: Anisotropy of the Taylor scale and the correlation scale in plasma sheet magnetic field fluctuations as a function of auroral electrojet activity, J. Geophys. Res., 115, A12250, doi:10.1029/2010JA015499, 2010.

Weygand, J. M., Matthaeus, W. H., Dasso, S., and Kivelson, M. G.: Correlation and Taylor scale variability in the interplanetary magnetic field fluctuations as a function of solar wind speed, J. Geophys. Res., 116, A08102, doi:10.1029/2011JA016621, 2011.

Wicks, R. T., Owens, M. J., and Horbury, T. S.: The variation of solar wind correlation lengths over three solar cycles, Solar Phys., 262, 191-198, doi:10.1007/s11207-010-9509-4, 2010. 